\title{
Septochoanal Polyp with Metaplastic Ossification Mimicking Sinonasal Tumor: A Case Report
}

\author{
Il-Young Cho, ${ }_{1}^{1}$ Jae-Wook Kim, ${ }^{1}$ Hak-Jin Kim, ${ }^{2}$ and Kyu-Sup Cho, ${ }^{1, *}$ \\ ${ }^{1}$ Department of Otorhinolaryngology and Biomedical Research Institute, Pusan National University School of Medicine, Pusan National University Hospital, Busan, Republic \\ of Korea \\ ${ }^{2}$ Department of Radiology, Pusan National University School of Medicine, Pusan National University Hospital, Busan, Republic of Korea \\ "Corresponding author: Kyu-Sup Cho, Department of Otorhinolaryngology and Biomedical Research Institute, Pusan National University School of Medicine, Pusan National \\ University Hospital, 179 Gudeok-Ro, Seo-gu, Busan 602-739, Republic of Korea. Tel: +82-512407824, Fax: +82-512468668, E-mail: choks@pusan.ac.kr
}

Received 2015 December 07; Revised 2016 February 06; Accepted 2016 February 15.

\begin{abstract}
Choanal polyps are a form of nasal polyps that grow toward the choana with a single stalk. Septochoanal polyp is a rare entity that originates from the mucosa of the nasal septum with choanal extension. The most common site of its origin is mainly the superior aspect of the posterior portion of the nasal septum. However, metaplastic ossification is a rare event in nasal polyp and to the best of our knowledge, septochoanal polyp with metaplastic ossification has not been reported in the literature. Herein, we report a case of huge septochoanal polyp with metaplastic ossification obstructing both the nasal cavity and nasopharynx, which was successfully removed via transnasal endoscopic approach.
\end{abstract}

Keywords: Nasal Polys, Nasal Septum, Heterotopic Ossification, Metaplasia, Nasal Obstruction

\section{Introduction}

Septochoanal polyp that originates from the mucosa of the nasal septum and extends into the choana is relatively rare $(1,2)$. Various histologic changes, such as epithelial atypia and squamous metaplasia may occur in the respiratory epithelium covering nasal polyps. However, metaplastic ossification of nasal polyps is extremely rare $(3,4)$. To the best of our knowledge, septochoanal polyp with metaplastic ossification has not been reported in the literature. Herein, we describe this rare clinical presentation of a huge septochoanal polyp with metaplastic ossification obstructing both the nasal cavity and nasopharynx. This case report was approved by the institutional review board of Pusan national university hospital.

\section{Case Presentation}

A 77-year-old man presented with gradually progressive nasal obstruction of both sides for 20 years. There was no history of nasal discharge and no sign of allergy. The endoscopic examination revealed obstruction of the posterior choanae by a huge mass of both nasal cavities filling the entire nasopharynx and extending into the oropharynx (Figure 1). A computed tomography (CT) scan of the paranasal sinuses showed a multi-lobulated soft tissue lesion with adjacent bony remodeling and diffuse irregular ossification in both posterior nasal cavities, the na- sopharynx, and oropharynx (Figure 2A-C). On magnetic resonance (MR) imaging, metaplastic ossification had heterogeneous high signal intensity on T1-weighted (T1W) images and low signal intensity on T2-weighted (T2W) images with mild reticular enhancement (Figure 2D-F). Haziness was also noted in frontal, ethmoidal, and maxillary sinuses. Endoscopic sinus surgery was performed under general anesthesia. The pedicle of the mass was identified on the right posterior nasal septum (Figure 3A). The base of the lesion including the healthy mucosa around it was removed and cauterized using suction cautery for prevention of recurrence. The mass was removed via the oropharynx (Figure 3B). Histopathologic examination showed a benign nasal polyp with extensive metaplastic bone formation in the stroma (Figure 4). According to the operative and histopathologic findings, this case was diagnosed as a septochoanal polyp with metaplastic ossification. The patient had an uneventful recovery and nasal obstruction disappeared. Endoscopic examinations performed 6 months postoperatively demonstrated no evidence of recurrence.

\section{Discussion}

Nasal polyps are the most common expansile lesions in the nasal cavity. They are formed by accumulation of fluid in the deeper lamina propria of the Schneiderian mucosa. Almost all nasal polyps originate from the mucosa of the lateral walls of the nasal cavity or from the paranasal 

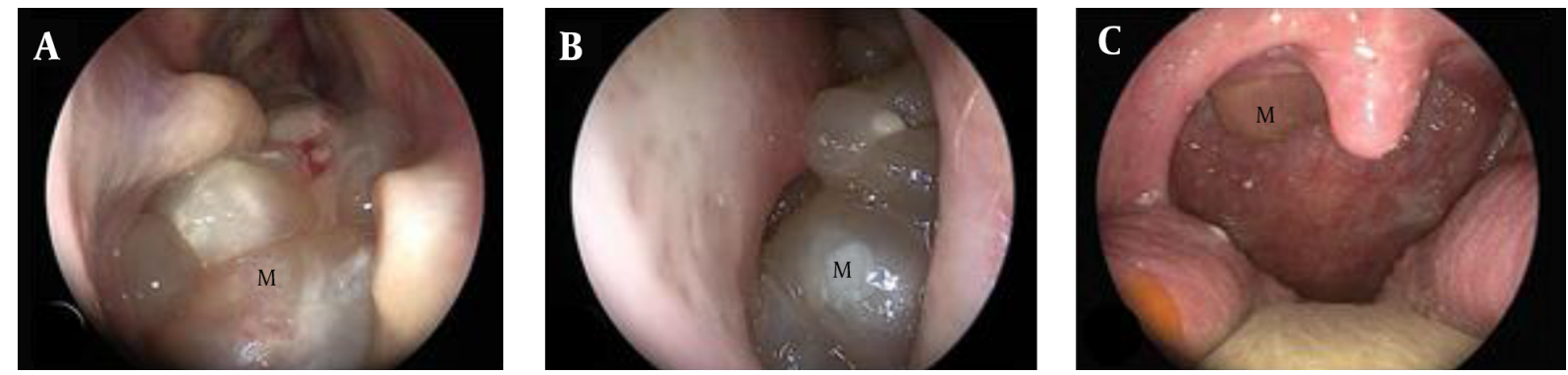

Figure 1. A 77-year-old man with gradually progressive nasal obstruction. A, Right and B, Left nasal endoscopy shows a huge polypoid mass (M) in the nasal cavity obstructing the posterior choanae; C, Oral cavity examination reveals the mass extending into the oropharynx.
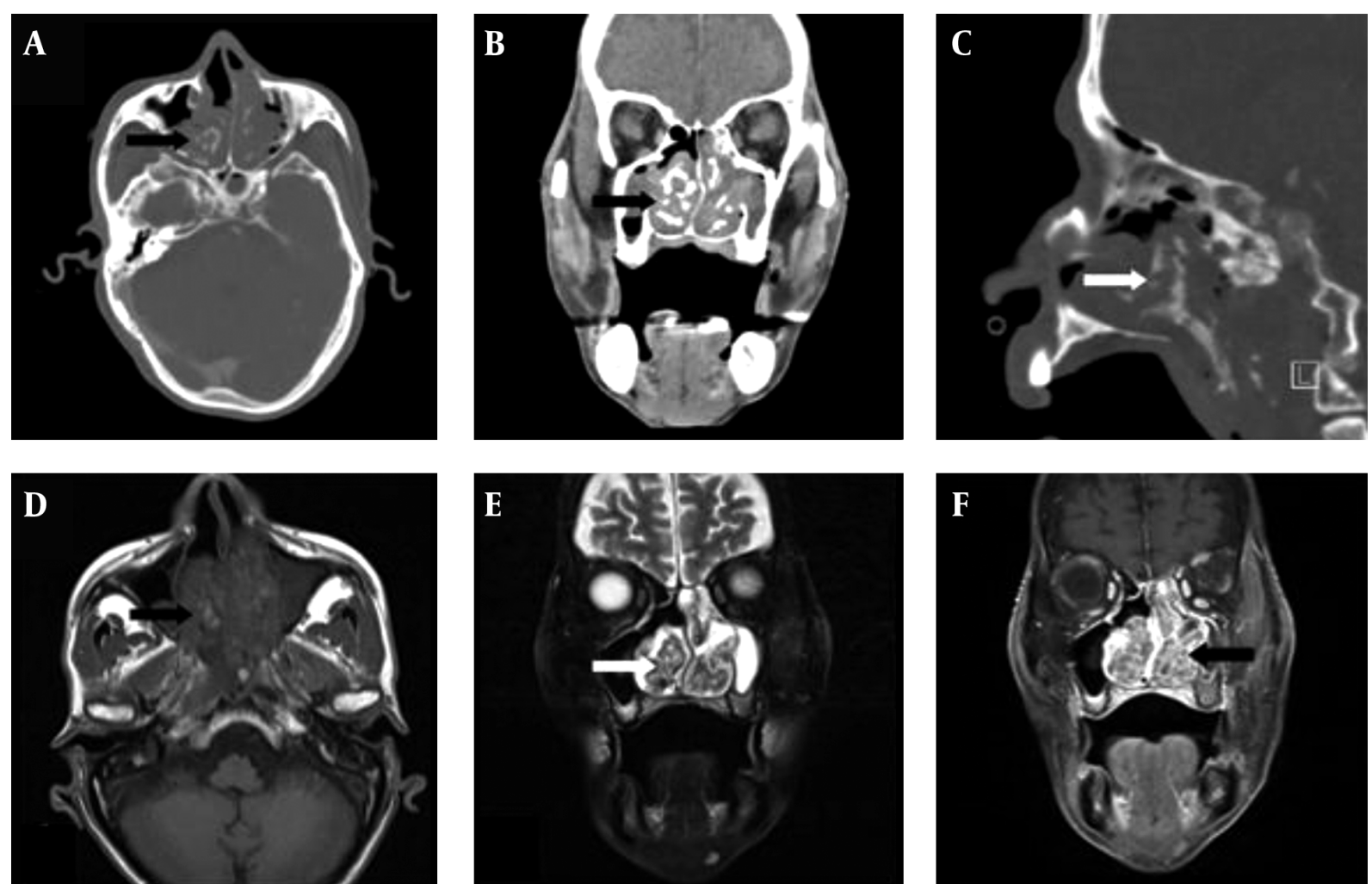

Figure 2. A-C, Enhanced CT of paranasal sinuses shows a large, lobulated mass with diffuse irregular ossification (arrows in A, B, and C) in the bilateral posterior nasal cavities, nasopharynx, and oropharynx; D-F, Metaplastic ossification shows heterogeneous high signal intensity on T1WI and low signal intensity on T2WI with mild reticular enhancement (arrows in D,E and F). Sinusitis is also seen in the left maxillary sinus as low density on CT, low signal intensity on T1WI, and strong high signal intensity on T2WI with no contrast enhancement.

sinuses (1). Septochoanal polyp is a rare entity that originates from the mucosa of the nasal septum with choanal extension. Septochoanal polyps are benign, unilateral, and invade the posterior nasal cavity through the nasopharynx (2). The most common site of their origin is mainly the superior aspect of the posterior portion of the nasal septum $(1,2,5)$. However, in our case, the polyp originated from the posteroinferior portion of the nasal septum with a thin pedicle and extended down to the oropharynx. Although heterotrophic bone formation (osseous metaplasia) has been described in many parts of the body, it is an extremely uncommon finding in nasal polyps. The pathogenesis of nasal polyp with metaplastic ossification is still unknown. It is presumed that pluripotent cells, which are either present in the stroma of the polyp or dedifferentiated from cells present in the polyp, may later differenti- 

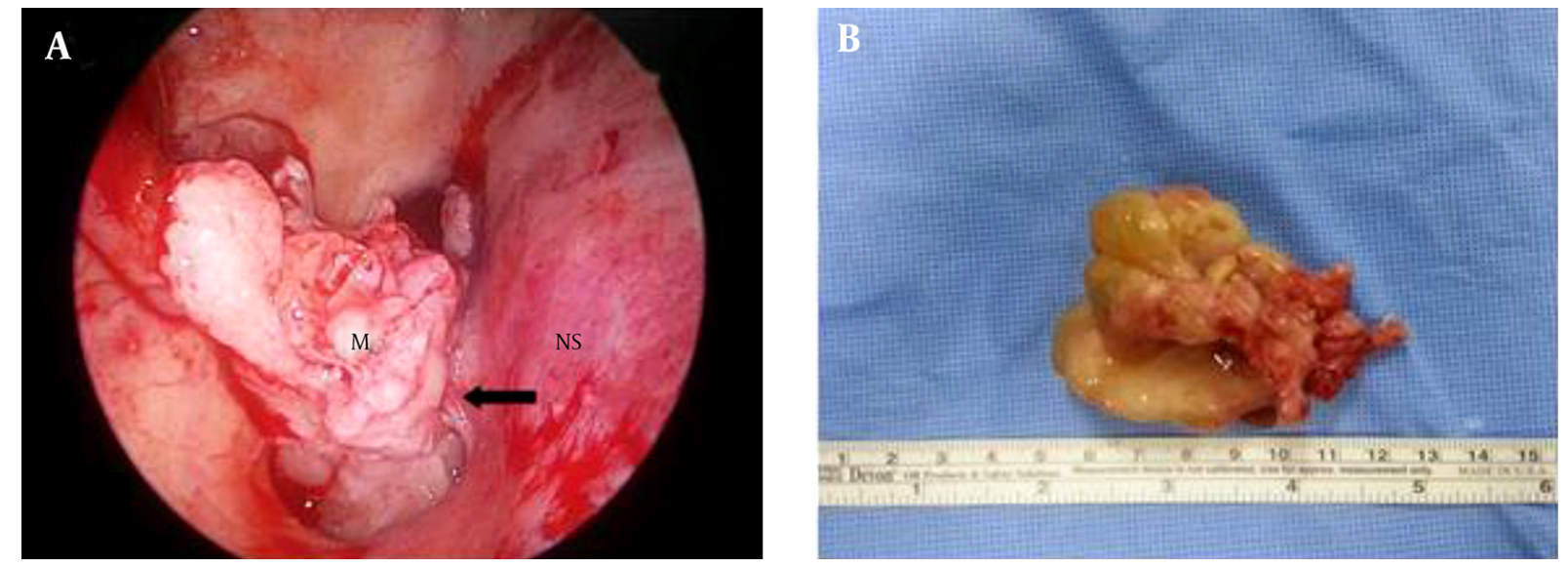

Figure 3. Intraoperative findings. A, The pedicle (arrow) of the mass (M) originates from the posteroinferior portion of the nasal septum (NS) and extends down to the oropharynx; B, A $5 \times 3 \times 7 \mathrm{~cm}$ sized mass is removed via the oropharynx.
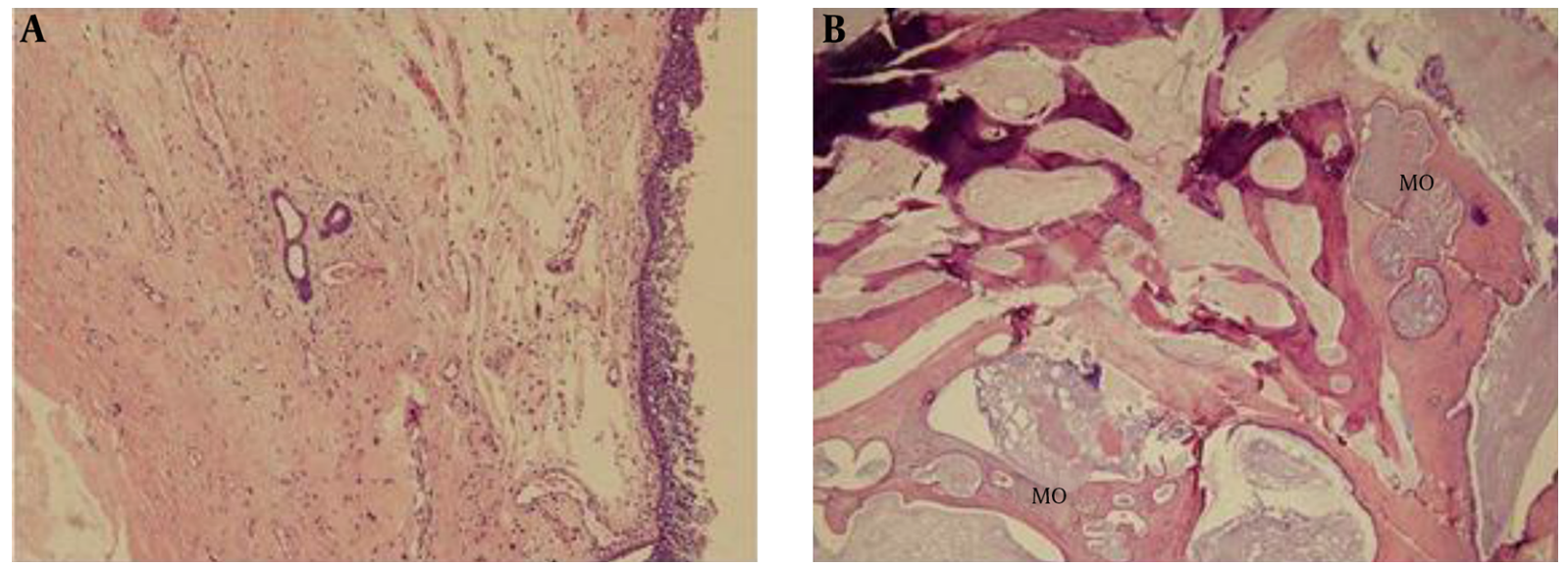

Figure 4. Histopathologic findings of septochoanal polyp with metaplastic ossification. A, The polyp is composed of a myxohyaline stroma covered by respiratory epithelium. It shows inflammatory cell infiltration and central degenerative change $(\mathrm{H} \& \mathrm{E}, \times 100)$; B, The polyp shows metaplastic ossification $(\mathrm{MO})(\mathrm{H} \& \mathrm{E}, \times 40)$.

ate along an osteoblastic lineage leading to ossification (3, $4,6)$.

The typical symptom of nasal polyp with metaplastic ossification is unilateral nasal obstruction. Occasionally, patients present with bilateral nasal obstruction, anosmia, and headache $(3,4)$. In this case, the patient complained of nasal obstruction on both sides, because nasopharynx was obstructed by a huge septochoanal polyp with metaplastic ossification.

Detection of the polyp origin by nasal endoscopy is important for the diagnosis of septochoanal polyp. However, radiologic examination is useful and essential for the diagnosis of septochoanal polyp with metaplastic ossification (7). A variety of pathologic conditions may mimic septochoanal polyp with metaplastic ossification on CT and MR images. These pathologies include nontumorous conditions such as rhinolith and fungal ball, and tumorous conditions, such as inverted papilloma, chondrosarcoma, osteosarcoma, and fibroosseous lesions (7).

The treatment of septochoanal polyp with metaplastic ossification is surgical removal via endoscopic approach. The septochoanal polyp may be completely removed by endoscopic surgery, and resection of a small amount of healthy mucosa surrounding the origin of the pedicle is necessary for preventing recurrence $(1,2,5)$.

Although septochoanal polyp is known to be very rare, it must be included in the differential diagnosis of nasal cavity mass. Furthermore, metaplastic ossification within septochoanal polyp should be considered in the benignlooking nasal cavity mass with centrally located radio- 
dense materials on CT scans.

\section{Footnotes}

Authors' Contributions: Il-Young Cho drafted the manuscript. Jae Wook Kim was responsible for acquisition of the data. Hak Jin Kim analyzed and interpreted the data. Kyu Sup Cho was responsible for study concept and design.

Financial Disclosure: None declared.

Funding/Support: This work was supported by the 2015 clinical research grant from Pusan national university hospital.

\section{References}

1. Ozgirgin ON, Kutluay L, Akkuzu G, Gungen Y. Choanal polyp originating from the nasal septum: a case report. Am J Otolaryngol. 2003;24(4):261-4. [PubMed: 12884221].
2. Cho HS, Kim KS. Nasal obstruction due to septochoanal polyp. Braz J Otorhinolaryngol. 2014;80(4):362-3. doi: 10.1016/j.bjorl.2014.05.024. [PubMed: 25183189].

3. Yilmaz M, Ibrahimov M, Kilic E, Ozturk O. Heterotopic bone formation (osseous metaplasia) in nasal polyps. J Craniofac Surg. 2012;23(2):620. doi: 10.1097/SCS.ob013e3182420592. [PubMed: 22446434].

4. Mercan H, Edizer DT, Kilic E, Esen T, Ramazanoglu R, Cansiz H. Osseous metaplasia in a nasal polyp: report of a rare case and review of the literature. Ear Nose Throat J. 2012;91(9):E4-6. [PubMed: 22996719].

5. Birkent H, Karahatay S, Durmaz A, Kurt B, Tosun F. Choanal polyp originating from the nasal septum: septochoanal polyp. Kulak Burun Bogaz Ihtis Derg. 2009;19(3):163-6. [PubMed: 19857197].

6. Jacono AA, Sclafani AP, Van De Water T, McCormick S, Frenz D. Metaplastic bone formation in nasal polyps with histologic presence of transforming growth factor beta-1 (TGFbeta-1) and bone morphogenetic proteins (BMPs). Otolaryngol Head Neck Surg. 2001;125(1):96-7. doi: 10.1067/mhn.2001.115857. [PubMed: 11458222].

7. Kim YK, Kim HJ, Kim J, Chung SK, Kim E, Ko YH, et al. Nasal polyps with metaplastic ossification: CT and MR imaging findings. Neuroradiology. 2010;52(12):1179-84. doi: 10.1007/s00234-010-0758-6. [PubMed: 20717820]. 